\title{
The Gray filtration on phantom maps
}

by

Lê Minh Hà (Toledo, OH) and Jeffrey Strom (Hanover, NH)

\begin{abstract}
This paper is a study of the Gray index of phantom maps. We give a new, tower theoretic, definition of the Gray index, which allows us to study the naturality properties of the Gray index in some detail.

McGibbon and Roitberg have shown that if $f^{*}$ is surjective on rational cohomology, then the induced map on phantom sets is also surjective. We show that if $f^{*}$ is surjective just in dimension $k$, then $f$ induces a surjection on a certain subquotient of the phantom set. If the condition holds for all $k$, we recover McGibbon and Roitberg's theorem. There is a dual result, and a theorem on phantom maps into spheres which holds one dimension at a time as well.

Finally, we examine the set of phantom maps whose Gray index is infinite. The main theorem is a partial verification of our conjecture that if $X$ and $Y$ are nilpotent and of finite type, then every phantom map $f: X \rightarrow Y$ must have finite index.
\end{abstract}

Introduction. A map $f: X \rightarrow Y$ from one $\mathrm{CW}$ complex to another is a phantom map if its restriction to the $k$-skeleton of $X,\left.f\right|_{X_{k}}$, is nullhomotopic for each $k$; this happens if and only if there are homotopy factorizations

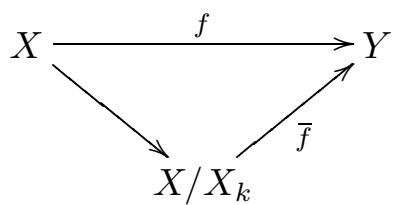

for each $k$. The map $\bar{f}$ is usually not uniquely determined in this diagram. The Gray index $G(f)$ of $f$ is the least integer $k$ for which the map $\bar{f}$ cannot be chosen to be a phantom map. The Gray index gives rise to a filtration on the set $\operatorname{Ph}(X, Y)$ of phantom maps from $X$ to $Y$, which we call the Gray filtration. Explicitly, we set $\operatorname{Ph}^{k}(X, Y)=\{f \in \operatorname{Ph}(X, Y) \mid G(f) \geq k\}$, so

$$
\operatorname{Ph}(X, Y)=\operatorname{Ph}^{1}(X, Y) \supseteq \operatorname{Ph}^{2}(X, Y) \supseteq \ldots \supseteq \mathrm{Ph}^{k}(X, Y) \supseteq \ldots
$$

2000 Mathematics Subject Classification: 55S37, 55P30, 55P62, 20 J05.

Key words and phrases: phantom maps, Gray index, inverse limit, $\lim ^{1}$. 
Our results concern this filtration and how it behaves under maps between domains or targets. In the introduction, all spaces will be finite type domains, or ranges as needed; see conventions at the end of this section.

Before summarizing the results of this paper, we provide some motivation. Brayton Gray observed in [3] that if there is an essential phantom map $f: X \rightarrow Y$, then there must be a dimension $n$ in which both $H^{n}(X ; \mathbb{Q}) \neq 0$ and $\pi_{n+1}(Y) \otimes \mathbb{Q} \neq 0$. The proof of this fact is entirely nonconstructive, so it leaves open an interesting, if ill-posed, question: which of the dimensions $n$ in which $H^{n}(X ; \mathbb{Q}) \neq 0$ and $\pi_{n+1}(Y) \otimes \mathbb{Q} \neq 0$ can be deduced from (is "relevant" to) a given phantom map $f$ ? We should expect two answers - if dimension $n$ is "relevant" to $f$ in terms of the domain, then dimension $n+1$ should be "relevant" to $f$ in terms of the target. The underlying intuitive idea of this paper is that the least dimension which is "relevant" to $f$ (in terms of the domain) is its Gray index, $G(f)$.

Now we illustrate how this intuitive idea suggests actual theorems. In [8], McGibbon and Roitberg prove that if $\phi: A \rightarrow B$ induces surjections on rational cohomology in all dimensions, then the induced map on phantom sets

$$
\phi^{*}: \operatorname{Ph}(B, Y) \rightarrow \operatorname{Ph}(A, Y)
$$

is also surjective for any finite type target $Y$. A careful look at the proof shows that the conclusion remains true if $\phi$ induces surjections just in those dimensions $n$ for which Gray's algebraic condition $H^{n}(X ; \mathbb{Q}) \neq 0$ and $\pi_{n+1}(Y) \otimes \mathbb{Q} \neq 0$ holds. It seems reasonable to expect that if $\phi$ is surjective only in those dimensions that are "relevant" to $f$, then $f$ should be in the image of $\phi^{*}: \operatorname{Ph}(B, Y) \rightarrow \operatorname{Ph}(A, Y)$. We actually prove a more delicate statement in the following theorem.

Theorem 4. If $\phi: A \rightarrow B$ induces surjections

$$
\phi^{*}: H^{m}(B ; \mathbb{Q}) \rightarrow H^{m}(A ; \mathbb{Q})
$$

in the range $k \leq m \leq l$ and $f \in \operatorname{Ph}(A, Y)$ with $k \leq G(f) \leq l$, then $f$ is in the image of $\phi^{*}: \operatorname{Ph}(B, Y) \rightarrow \operatorname{Ph}(A, Y)$, modulo $\mathrm{Ph}^{l+1}(A, Y)$.

In particular, if $\phi$ induces a surjection in rational cohomology just in dimension $G(f)$, then $f$ is in the image of $\phi^{*}: \operatorname{Ph}(B, Y) \rightarrow \operatorname{Ph}(A, Y)$, modulo an indeterminacy which does not contain $f$. McGibbon and Roitberg prove a dual result for maps between targets; our refinement also dualizes.

In Theorem 1 of [8], McGibbon and Roitberg show that for finite type nilpotent $X$, the conditions

(a) $\operatorname{Ph}\left(X, S^{k+1}\right)=*$ for all $k$,

(b) there is a map $X \stackrel{g}{\longrightarrow} \bigvee S^{n_{\alpha}}$ such that

$$
g^{*}: H^{*}\left(\bigvee S^{n_{\alpha}} ; \mathbb{Q}\right) \rightarrow H^{*}(X ; \mathbb{Q})
$$

is an isomorphism, 
(c) $\operatorname{Ph}(X, Y)=*$ for all finite type nilpotent $Y$,

are equivalent. Using the Gray index, we show that this result is actually a reflection of a feature of phantom maps that holds in one dimension at a time.

THEOREM 8. Let $X$ be nilpotent and of finite type. Each of the following statements implies the next:

(1) $\operatorname{Ph}\left(X, S^{k+1}\right)=*$;

(2) there is a map $X \stackrel{g}{\longrightarrow} \prod \Omega S^{k+1}$ such that

$$
g^{*}: H^{k}\left(\prod \Omega S^{k+1} ; \mathbb{Q}\right) \rightarrow H^{k}(X ; \mathbb{Q})
$$

is surjective;

(3) $\mathrm{Ph}^{k}(X, Y)=\mathrm{Ph}^{k+1}(X, Y)$ for all finite type nilpotent $Y$.

Again, our result dualizes.

In Section 4, we study phantom maps with infinite Gray index. If $G(f)=$ $\infty$ then none of the dimensions in which $H^{n}(X ; \mathbb{Q}) \neq 0$ and $\pi_{n+1}(Y) \otimes \mathbb{Q} \neq 0$ (whose existence is guaranteed by $f$ ) is "relevant" to $f$, which suggests the following conjecture.

ConjeCture. If $X$ and $Y$ are both nilpotent and of finite type, then $\operatorname{Ph}^{\infty}(X, Y)=*$.

We do not prove this conjecture here. However, we do have some partial results which show that, in many situations, the phantoms with infinite index (if they exist) take care of themselves. Our main theorem in Section 4 is the following.

TheOrem 10. Suppose $X$ and $Y$ are of finite type and that every phantom map from $X$ to $Y$ has infinite index. Then $\operatorname{Ph}(X, Y)=*$.

Conventions. Finally, we quickly review some definitions and notation that we will use throughout. All the spaces in this paper have the homotopy type of CW complexes. The $n$-skeleton of a CW complex $X$ is denoted by $X_{n}$. We use $X^{(n)}$ to denote the $n$th Postnikov section of $X$, and $X\langle n\rangle$ to denote the $n$th connective cover of $X$. Thus, there are natural fibrations

$$
X\langle n\rangle \rightarrow X \rightarrow X^{(n)} .
$$

For many of the results in this paper we need to impose finiteness conditions on our spaces. We follow McGibbon and Roitberg [8] in defining a finite type domain to be a pointed connected CW complex whose integral homology groups are finitely generated in each degree, and a finite type target to be a pointed space each of whose homotopy groups is finitely generated.

Acknowledgements. Before we begin, we would like to express our thanks to Chuck McGibbon for his help and encouragement as we have 
learned about phantom maps. We would also like to thank Kouyemon Iriye for pointing out a mistake in one of our examples.

1. The Gray index. The Gray index was first defined by Gray in his thesis [3]; it has also been studied by McGibbon and Strom in [10]. It is important to note that our definition differs by one from Gray's original definition [3] (ours is bigger). Let $f: X \rightarrow Y$ be a phantom map. Then $\left.f\right|_{X_{k}} \simeq *$ for each $k$, and so there are homotopy factorizations of $f$

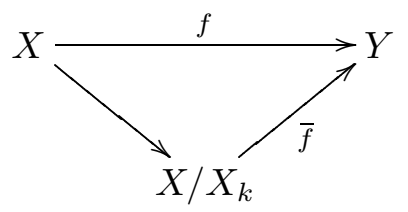

Generally, there will be many possible choices for $\bar{f}$.

Definition. The Gray index of $f$ is the least integer $k$ such that the map $\bar{f}$ cannot be chosen to be a phantom map; we write $G(f)=k$. If there is no such integer, then $G(f)=\infty$.

We denote the set of all phantom maps $f: X \rightarrow Y$ with $G(f) \geq k$ by $\mathrm{Ph}^{k}(X, Y)$. Thus, $\mathrm{Ph}^{k+1}(X, Y)=\operatorname{Im}\left(\operatorname{Ph}\left(X / X_{k}, Y\right) \rightarrow \operatorname{Ph}(X, Y)\right)$. It follows from Proposition 1 below that this definition is independent of the CW decomposition of $X$.

If $f: X \rightarrow Y$ is a phantom map, then the image of $f$ must be contained in the basepoint component of $Y$, or else the restriction of $f$ to the 0 -skeleton of $X$ will be essential. Also, $f$ must remain phantom when restricted to each component of $X$. If we give $X$ a CW decomposition with one 0 -cell in each component, we see that $f$ factors through a phantom map $X / X_{0} \rightarrow Y$, and so $\operatorname{Ph}(X, Y)=\mathrm{Ph}^{1}(X, Y)$.

There is a dual definition of phantom maps in terms of the connective covers of the target. A map $f: X \rightarrow Y$ is phantom if and only if $f$ factors (up to homotopy) as in the diagram

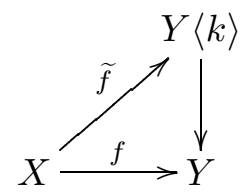

for each $k \geq 0$. We can then define a dual Gray index, $G^{\prime}(f)$, to be the least integer $k$ such that the map $\tilde{f}$ cannot be chosen to be a phantom map.

Intuitively, $G^{\prime}(f)$ is the least dimension "relevant" to $f$ in terms of the target. In view of Gray's observation we expect that the least "relevant" dimension for the target should be one more than the least "relevant" dimension for the domain. This is in fact the case. 
Proposition 1. If $f: X \rightarrow Y$ is a phantom map, then $G^{\prime}(f)=G(f)+1$.

Proofs will be given in the last section.

It follows that $\mathrm{Ph}^{k}(X, Y)=\operatorname{Im}(\operatorname{Ph}(X, Y\langle k\rangle) \rightarrow \operatorname{Ph}(X, Y))$. This result also shows that $G(f)$ is independent of any choices that were made in giving $X$ a $\mathrm{CW}$ decomposition.

We will make essential use of the following alternative description of the Gray index in terms of inverse towers. According to Bousfield and Kan ([1], pages 254-255), $\mathrm{Ph}(X, Y)$ is naturally isomorphic to a $\lim ^{1}$ set:

$$
\operatorname{Ph}(X, Y) \cong \lim _{n}^{1}\left[X, \Omega Y^{(n)}\right]
$$

Observe that in the notation $\Omega Y^{(n)}$ the order of operations is ambiguous. In this paper, as in most other papers about phantom maps, passing to the loop space is the last step. Thus, $\Omega Y^{(n)}$ really means $\Omega\left(Y^{(n)}\right)$. Let us write $G_{n}=\left[X, \Omega Y^{(n)}\right]$ and

$$
G_{k}^{(n)}=\operatorname{Im}\left(G_{n} \rightarrow G_{k}\right) .
$$

We have a commutative diagram of surjections of towers

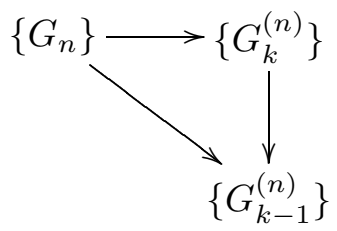

which induces a commutative diagram of surjections after taking $\lim ^{1}$

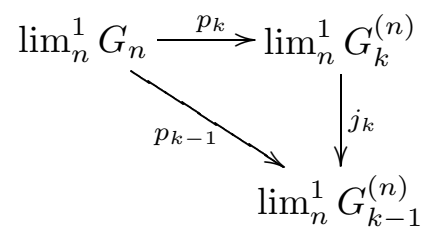

The maps $p_{k}$ define equivalence relations on $\operatorname{Ph}(X, Y)$ : say $f \sim_{k} g$ if and only if $p_{k}(f)=p_{k}(g)$. The commutativity of the diagram shows that $f \sim_{k} g$ implies $f \sim_{k-1} g$, but the reverse need not be true. We interpret $\lim _{n}^{1} G_{k}^{(n)}$ as the set of $\sim_{k^{-}}$equivalence classes of $\operatorname{Ph}(X, Y)$.

TheOREM 2. Let $f: X \rightarrow Y$ be a phantom map. Then $G^{\prime}(f)$ is the least integer $k$ such that $f \chi_{k} *$, and $G(f)$ is the greatest integer $k$ such that $f \sim_{k} *$.

In other words, we have $\operatorname{Ph}^{k}(X, Y)=\operatorname{Ker}\left(p_{k}\right)$. These sets give us a natural filtration

$$
\operatorname{Ph}(X, Y)=\operatorname{Ph}^{1}(X, Y) \supseteq \operatorname{Ph}^{2}(X, Y) \supseteq \ldots \supseteq \mathrm{Ph}^{k}(X, Y) \supseteq \ldots
$$

which we call the Gray filtration. 
Typically one studies a filtered group by examining the subquotients. Unfortunately, the pointed set $\operatorname{Ph}(X, Y)$ does not generally have a group structure. Nevertheless, we can use the equivalence relations $\sim_{k}$ to make sense of subquotients of this filtration. Write

$$
\mathrm{Ph}^{k}(X, Y) / \mathrm{Ph}^{l}(X, Y)=p_{l}\left(\operatorname{Ph}^{k}(X, Y)\right) \subseteq \lim _{n}^{1} G_{l}^{(n)} .
$$

Clearly, this definition agrees with the usual notion whenever $\operatorname{Ph}(X, Y)$ happens to be a group.

Our final result in this section is that the Gray filtration can only decrease if Gray's algebraic condition $H^{n}(X ; \mathbb{Q}) \neq 0$ and $\pi_{n+1}(Y) \otimes \mathbb{Q} \neq 0$ is met.

Proposition 3. Assume $X$ and $Y$ are nilpotent spaces of finite type. If either $H^{k}(X ; \mathbb{Q})=0$ or $\pi_{k+1}(Y) \otimes \mathbb{Q}=0$, then

$$
\mathrm{Ph}^{k}(X, Y)=\mathrm{Ph}^{k+1}(X, Y) \text {. }
$$

This means that if either $H^{*}(X ; \mathbb{Q})$ is bounded above by $n$ or $\pi_{*}(Y) \otimes \mathbb{Q}$ is bounded above by $n+1$, then the Gray filtration is finite. More precisely, $\mathrm{Ph}^{n}(X, Y)=\mathrm{Ph}^{\infty}(X, Y)$; we will see in Corollary 11 below that this implies that $\operatorname{Ph}^{n}(X, Y)=*$. On the other hand, if either $H^{*}(X ; \mathbb{Q})$ is bounded below by $n$ or $\pi_{*}(Y) \otimes \mathbb{Q}$ is bounded below by $n+1$, then every phantom map $f: X \rightarrow Y$ has $G(f) \geq n$.

Example. Consider the Gray index of phantom maps $X \rightarrow S^{k+1}$. For any $k$, we have

$$
\operatorname{Ph}\left(X, S^{k+1}\right)=\operatorname{Ph}^{k}\left(X, S^{k+1}\right) .
$$

If $k$ is even, then $S^{k+1}$ has only one nontrivial rational homotopy group, and so

$$
\operatorname{Ph}^{k+1}\left(X, S^{k+1}\right)=* .
$$

If $k$ is odd, then there are two dimensions to consider, so

$$
\mathrm{Ph}^{k+1}\left(X, S^{k+1}\right)=\mathrm{Ph}^{2 k}\left(X, S^{k+1}\right) \quad \text { and } \quad \mathrm{Ph}^{2 k+1}\left(X, S^{k+1}\right)=* .
$$

2. Maps subject to rational conditions. Now we turn our attention to the problem of determining how the Gray filtration behaves with respect to maps between targets or domains.

Theorem 4. Let $A$ and $B$ be finite type domains and $Y$ a finite type target. If $\phi: A \rightarrow B$ induces surjections $\phi^{*}: H^{m}(B ; \mathbb{Q}) \rightarrow H^{m}(A ; \mathbb{Q})$ in the range $k \leq m \leq l$ then

$$
p_{l+1} \phi^{*}\left(\mathrm{Ph}^{k}(B, Y)\right)=\mathrm{Ph}^{k}(A, Y) / \mathrm{Ph}^{l+1}(A, Y) .
$$

Suppose $\phi$ induces a rational cohomology surjection only in dimension $k$. Then Theorem 4 says that if $G(f)=k$ (so $f \not_{k+1} *$ ), then there is a phantom map $f^{\prime} \sim_{k+1} f$ such that $f^{\prime}$ is in the image of $\phi^{*}$. In particular, the map $\phi^{*}:[B, Y] \rightarrow[A, Y]$ is nontrivial. Here is a concrete example. 
EXAMPLE. There are phantom maps $\mathbb{C P}^{\infty} \rightarrow S^{2} \vee S^{2}$ of every even Gray index [10]. Therefore, if $f: \mathbb{C P}^{\infty} \rightarrow Y$ is a map which is nonzero in rational cohomology, then our discussion applies, showing that the induced map

$$
\left[Y, S^{2} \vee S^{2}\right] \stackrel{f^{*}}{\longrightarrow}\left[\mathbb{C P}^{\infty}, S^{2} \vee S^{2}\right]
$$

must be nontrivial.

The following example shows how Theorem 4 can be used to construct exact sequences of phantom sets.

ExAmple. Let $f: X \rightarrow S^{2 n}$ be an essential phantom map. If $\Sigma f \not *$, then the composition

$$
X \stackrel{f}{\longrightarrow} S^{2 n} \stackrel{\sigma}{\longrightarrow} \Omega S^{2 n+1}
$$

is nontrivial, and it follows that $G(f)=2 n-1$. If $\Sigma f \simeq *$ then there is a map $\tilde{f}: X \rightarrow S^{2 n}\langle 4 n-2\rangle$ lifting $f$ as in the diagram

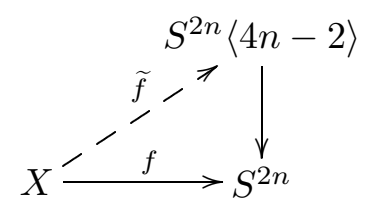

In other words, there is an exact sequence of pointed sets

$$
\left[X, S^{2 n}\langle 4 n-2\rangle\right] \rightarrow \operatorname{Ph}\left(X, S^{2 n}\right) \rightarrow \operatorname{Ph}\left(X, \Omega S^{2 n+1}\right) \rightarrow * .
$$

The surjectivity of $\mathrm{Ph}\left(X, S^{2 n}\right) \rightarrow \operatorname{Ph}\left(X, \Omega S^{2 n+1}\right)$ follows from Theorem 4 and Corollary 11 below.

It should be expected that we will get a dual version of Theorem 4, and we do.

Theorem 5. Let $A$ be a finite type domain and $Y$ and $Z$ be finite type targets. If $\phi: Y \rightarrow Z$ induces surjections $\pi_{m}(Y) \otimes \mathbb{Q} \rightarrow \pi_{m}(Z) \otimes \mathbb{Q}$ in the range $k \leq m \leq l$, then

$$
p_{l} \phi^{*}\left(\mathrm{Ph}^{k-1}(A, Y)\right)=\mathrm{Ph}^{k-1}(A, Z) / \mathrm{Ph}^{l}(A, Z) .
$$

In particular, if $f: A \rightarrow Z$ is a phantom map with $G(f)=k$ (so $\left.f \chi_{k+1} *\right)$ and $\phi_{*}$ is surjective in dimension $k+1$, then there is $f^{\prime} \sim_{k+1} f$ such that $f^{\prime} \in \phi^{*}(\operatorname{Ph}(A, Y))$.

Fibrations and cofibrations do not give rise to exact sequences of phantom maps. However, the composite of the maps induced by a cofibration or a fibration must be trivial. This allows us to pass from Theorems 4 and 5, which tell us how nearly surjective induced maps are, to Theorem 6 , which tells us how nearly trivial induced maps are.

Theorem 6. Let $\phi: A \rightarrow B$ be a map between finite type domains, and $Y$ a finite type target. Suppose that $\phi^{*}: H^{m}(B ; \mathbb{Q}) \rightarrow H^{m}(A ; \mathbb{Q})$ is zero for 
$k \leq m \leq l$. Then

$$
\phi^{*}\left(\mathrm{Ph}^{k}(B, Y)\right) \subseteq \mathrm{Ph}^{l+1}(A, Y) .
$$

Dually, if $\phi: Y \rightarrow Z$ and $\phi_{*}: \pi_{m}(Y) \otimes \mathbb{Q} \rightarrow \pi_{m}(Z) \otimes \mathbb{Q}$ is zero for $k \leq m \leq l$ then

$$
\phi_{*}\left(\mathrm{Ph}^{k-1}(A, Y)\right) \subseteq \mathrm{Ph}^{l}(A, Z) .
$$

We conclude this section by using the Gray index to put a topology on the set $\operatorname{Ph}(X, Y)$.

EXAMPLE 7. The sets $U_{k}(f)=\left\{g \mid g \sim_{k} f\right\}$ form the basis for a topology on $\mathrm{Ph}(X, Y)$. Observe that, in this topology, $\mathrm{Ph}^{\infty}(X, Y)$ is precisely the closure of the singleton set consisting of the trivial map. If $\mathrm{Ph}^{\infty}(X, Y)=*$, then the topology is Hausdorff.

Since $\phi_{l}: X \rightarrow X^{(l)}$ is an $(l-1)$-equivalence, Theorem 4 shows that

$$
\phi_{l}^{*}\left(\operatorname{Ph}\left(X^{(l)}, Y\right)\right) \cap U_{l}(f) \neq \emptyset .
$$

In other words, the union of the $\phi_{l}^{*}\left(\operatorname{Ph}\left(X^{(l)}, Y\right)\right)$ is dense in $\operatorname{Ph}(X, Y)$.

Suppose that $X$ is a finite type domain and that $Y$ is a finite complex. A theorem of Zabrodsky [13] shows that $\left[X^{(l)}, Y\right]=\operatorname{Ph}\left(X^{(l)}, Y\right)$. The commutativity of the diagram

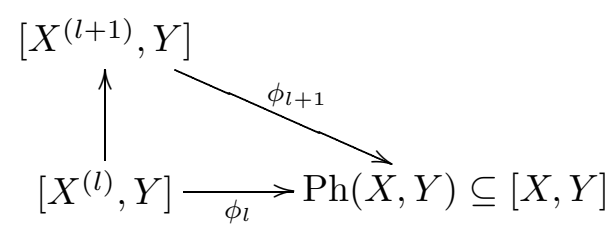

gives us a map $\operatorname{colim}_{l}\left[X^{(l)}, Y\right] \rightarrow \operatorname{Ph}(X, Y)$. The above discussion shows that the image of this map is dense in $\operatorname{Ph}(X, Y)$. Or, if we take the closure of the image, we find that

$$
\operatorname{Ph}(X, Y)=\overline{\operatorname{Im}\left(\operatorname{colim}_{l}\left[X^{(l)}, Y\right]\right)} .
$$

3. Phantom maps into spheres. In Theorem 1 of [8], McGibbon and Roitberg show that for finite type domains $X$, the conditions

(a) $\operatorname{Ph}\left(X, S^{k+1}\right)=*$ for all $k$,

(b) there is a map $X \stackrel{g}{\longrightarrow} \bigvee S^{n_{\alpha}}$ such that $g^{*}: H^{*}\left(\bigvee S^{n_{\alpha}} ; \mathbb{Q}\right) \rightarrow$ $H^{*}(X ; \mathbb{Q})$ is an isomorphism,

(c) $\operatorname{Ph}(X, Y)=*$ for all finite type targets $Y$,

are equivalent. This result is actually a reflection of a feature of phantom maps that holds in one dimension at a time.

TheOrem 8. Let $X$ be a finite type domain. Each of the following statements implies the next:

(1) $\operatorname{Ph}\left(X, S^{k+1}\right)=*$; 
(2) there is a map $X \stackrel{g}{\longrightarrow} \prod \Omega S^{k+1}$ such that $g^{*}: H^{k}\left(\prod \Omega S^{k+1} ; \mathbb{Q}\right) \rightarrow$ $H^{k}(X ; \mathbb{Q})$ is surjective;

(3) $\mathrm{Ph}^{k}(X, Y)=\mathrm{Ph}^{k+1}(X, Y)$ for all finite type targets $Y$.

It is reasonable to ask whether condition (3) implies condition (1) in our theorem. If $k$ is even, the answer is yes, because

$$
\mathrm{Ph}^{k}\left(X, S^{k+1}\right)=\mathrm{Ph}^{k+1}\left(X, S^{k+1}\right)=* .
$$

If $k$ is odd, however, the implication is not generally true, because

$$
\mathrm{Ph}^{k}\left(X, S^{k+1}\right)=\mathrm{Ph}^{2 k}\left(X, S^{k+1}\right),
$$

which need not be trivial, as the next example shows.

ExAmple. There are no essential phantom maps $f: \mathbb{C P}^{\infty} \rightarrow S^{4}$ with $G(f)=3$ (because $H^{3}\left(\mathbb{C P}^{\infty} ; \mathbb{Q}\right)=0$ ). However, there are essential phantom maps $\mathbb{C P}^{\infty} \rightarrow S^{4}$ with $G(f)=6$, because, according to Zabrodsky [13],

$$
\mathrm{Ph}\left(\mathbb{C P}^{\infty}, S^{4}\right)=\left[\mathbb{C P}^{\infty}, S^{4}\right] \cong\left[\mathbb{C P}_{0}^{\infty}, S^{4}\right] \cong H^{6}\left(\mathbb{C P}^{\infty} ; \pi_{7}\left(S^{4}\right) \otimes \mathbb{R}\right) \neq 0,
$$

where $\mathbb{R}$ is a rational vector space with the cardinality of the real numbers.

Theorem 8 implies Theorem 1 of [8]. If condition (1) is true for all $k$, then we find that $\operatorname{Ph}(X, Y)=\mathrm{Ph}^{\infty}(X, Y)$, which, by Theorem 10 below, means that $\operatorname{Ph}(X, Y)=*$ for all finite type $Y$, which in turn implies that condition (1) holds for all $k$. To recover the precise statement of McGibbon and Roitberg's result, suspend our map $g$ to obtain a map

$$
\Sigma X \rightarrow \bigvee_{i=1}^{m} S^{k+1} \vee \bigvee S^{n_{\alpha}}
$$

which induces a surjection in $H^{k+1}(-; \mathbb{Q})$. It is a simple matter to pinch off the irrelevant spheres and so obtain a map $\Sigma X \rightarrow \bigvee S^{k+1}$ which induces an isomorphism in $H^{k+1}(-; \mathbb{Q})$.

We end this section with the dual to Theorem 8 .

TheOREM 9. Let $Y$ be a finite type target. Each of the following statements implies the next:

(1) $\operatorname{Ph}(K(\mathbb{Z}, k), Y)=*$;

(2) there is a map $\bigvee \Sigma K(\mathbb{Z}, k) \stackrel{g}{\longrightarrow} Y$ such that

$$
\left.g^{*}: \pi_{k+1}(\bigvee \Sigma K(\mathbb{Z}, k)) \otimes \mathbb{Q}\right) \rightarrow \pi_{n+1}(Y) \otimes \mathbb{Q} \quad \text { is surjective; }
$$

(3) $\mathrm{Ph}^{k}(X, Y)=\mathrm{Ph}^{k+1}(X, Y)$ for all finite type domains $X$.

The deduction of McGibbon and Roitberg's Theorem $1^{\prime}$ from this result proceeds just as above. 
4. Maps with infinite Gray index. In his thesis [3], Gray claims that $G(f)<\infty$ for every essential phantom map $f$. However, there is a flaw in his argument; in fact, McGibbon and Strom [10] have shown that if $X$ is of finite type and its cohomology contains an element of infinite height under the action of the Steenrod algebra, then there are essential phantom maps out of $X$ with infinite Gray index.

In these examples, even though the domains are of finite type, the targets are definitely not. Thus, we make the following conjecture.

Conjecture. If $X$ is a finite type domain and $Y$ is a finite type target, then $\mathrm{Ph}^{\infty}(X, Y)=*$.

We have not been able to prove this conjecture, but we do have a useful partial result. Let $G_{n}=\left[X, \Omega Y^{(n)}\right]$ as in Section 1, and recall that a map $f \in \operatorname{Ph}(X, Y) \cong \lim _{n}^{1} G_{n}$ has infinite Gray index if and only if it is in the kernel of each map $p_{k}: \lim _{n}^{1} G_{n} \rightarrow \lim _{n}^{1} G_{k}^{(n)}$. The maps $p_{k}$ fit together to give us a surjective map $p_{\infty}: \operatorname{Ph}(X, Y) \rightarrow \lim _{k} \lim _{n}^{1} G_{k}^{(n)}$. Since $f$ has infinite Gray index if and only if it is in the kernel of $p_{\infty}$, we call the target of this map $\mathrm{Ph}(X, Y) / \mathrm{Ph}^{\infty}(X, Y)$. We will also use the notation

$$
\underline{\mathrm{Ph}}(X, Y)=\mathrm{Ph}(X, Y) / \mathrm{Ph}^{\infty}(X, Y) .
$$

Similarly, we will let $\underline{\mathrm{Ph}}^{k}(X, Y)=\mathrm{Ph}^{k}(X, Y) / \mathrm{Ph}^{\infty}(X, Y)$. Thus our conjecture is that $\operatorname{Ph}(X, Y)=\underline{\mathrm{Ph}}(X, Y)$ whenever both $X$ and $Y$ are nilpotent and of finite type.

Theorem 10. Let $X$ be a finite type domain and $Y$ a finite type target. If $\underline{\operatorname{Ph}}(X, Y)=*$, then $\operatorname{Ph}(X, Y)=*$.

In other words, the only way that $\operatorname{Ph}(X, Y)$ can consist solely of phantom maps with infinite Gray index is for $\operatorname{Ph}(X, Y)=*$.

Corollary 11. If $H^{m}(X, \mathbb{Q})=0$ or $\pi_{m+1}(Y) \otimes \mathbb{Q}=0$ for $m>k$, then

$$
\mathrm{Ph}^{k}(X, Y)=* \text {. }
$$

We can say much more if the towers we are concerned with happen to be towers of abelian groups.

Theorem 12. Let $A$ and $B$ be finite type domains and $Y$ and $Z$ be finite type targets. A map $\phi: A \rightarrow B$ induces a surjection

$$
\phi^{*}: \underline{\mathrm{Ph}}(B, \Omega Y) \rightarrow \underline{\mathrm{Ph}}(A, \Omega Y)
$$

if and only if it induces a surjection $\phi^{*}: \operatorname{Ph}(B, \Omega Y) \rightarrow \operatorname{Ph}(A, \Omega Y)$. Dually, a map $\phi: Y \rightarrow Z$ induces a surjection

$$
\phi_{*}: \underline{\mathrm{Ph}}(\Sigma A, Y) \rightarrow \underline{\mathrm{Ph}}(\Sigma A, Z)
$$

if and only if $\phi_{*}: \operatorname{Ph}(\Sigma A, Y) \rightarrow \operatorname{Ph}(\Sigma A, Z)$ is surjective. 
This theorem, like Theorem 10, is really a topological interpretation of an algebraic result on maps between towers. In our proof, we show that the algebraic proposition underlying Theorem 10 applies to the tower $\left\{C_{n}\right\}$, where $C_{n}$ is the cokernel of the map $\left[\Sigma B_{n}, \Omega Y\right] \rightarrow\left[\Sigma A_{n}, \Omega Y\right]$. In order to do this, we need the tower $\left\{C_{n}\right\}$ to be a tower of groups, which is why we require the target to be a loop space.

Recently, Lê Minh Hà has succeeded in proving that a map between "good" towers of groups induces a surjection on $\lim ^{1}$ if and only if the induced map on the abelianizations of the towers induces a surjection on $\lim ^{1}$ (see [5]). One application of this is a proof of Theorem 12 without the assumption that the target be a loop space.

Finally, let us see what we can derive when we take $l=\infty$ in Theorems 4 and 5 .

Corollary 13. If $\phi$ induces surjections $\phi^{*}: H^{m}(B ; \mathbb{Q}) \rightarrow H^{m}(A ; \mathbb{Q})$ for all $m \geq k$, then

$$
\underline{\mathrm{Ph}}^{k}(A, Y)=\phi^{*}\left(\underline{\mathrm{Ph}}^{k}(B, Y)\right) .
$$

If $Y=\Omega Y^{\prime}$, then we may replace $\underline{\mathrm{Ph}}$ with $\mathrm{Ph}$. Dually, if $\phi: Y \rightarrow Z$ induces surjections $\pi_{m}(Y) \otimes \mathbb{Q} \rightarrow \pi_{m}(Z) \otimes \mathbb{Q}$ in the range $k \leq m$, then

$$
\underline{\mathrm{Ph}}^{k-1}(A, Z)=\phi^{*}\left(\underline{\mathrm{Ph}}^{k-1}(A, Y)\right) \text {. }
$$

If $X=\Sigma X^{\prime}$, then we may replace $\underline{\mathrm{Ph}}$ with $\mathrm{Ph}$.

If the conjecture were known to be true, we would recover Theorem 2 of [8] by setting $k=0$ in this corollary. When $Y$ is a loop space or $X$ is a suspension, we do not need to appeal to the conjecture.

Taking $l=\infty$ in Theorem 6, we find that if $\phi: A \rightarrow B$ induces trivial maps in rational cohomology in dimensions greater than $k$, then $\phi^{*}\left(\mathrm{Ph}^{k}(B, Y)\right) \subseteq \mathrm{Ph}^{\infty}(A, Y)$. In other words,

$$
\phi^{*}\left(\underline{\mathrm{Ph}}^{k}(B, Y)\right)=* \subseteq \underline{\mathrm{Ph}}(A, Y) .
$$

The (possibly) stronger conclusion $\phi^{*}(\mathrm{Ph}(X, Y))=*$ is also true; it follows from Theorem 2 of [8].

\section{Proofs}

Proof of Proposition 1. This proof uses the identification $\operatorname{Ph}(X, Y) \cong$ $\lim _{n}^{1}\left[X, \Omega Y^{(n)}\right]$ discussed immediately following the statement of Proposition 1. First suppose that $G(f)>k$. This means that there is a phantom map $\bar{f}: X / X_{k} \rightarrow Y$ extending $f$. We will show that $\bar{f}$ lifts to a phantom map $\widetilde{f}: X / X_{k} \rightarrow Y\langle k+1\rangle$. Indeed, the map of towers (with $k$ fixed and $n$ variable)

$$
\left\{\left[X / X_{k}, \Omega Y^{(n)}\langle k+1\rangle\right]\right\} \rightarrow\left\{\left[X / X_{k}, \Omega Y^{(n)}\right]\right\}
$$


is surjective. Since $\lim ^{1}$ is right exact [1], the induced map on phantom sets is surjective as well.

To go in the other direction, observe that the map of towers

$$
\left\{\left[X / X_{k}, \Omega Y^{(n)}\langle k+1\rangle\right]\right\} \rightarrow\left\{\left[X, \Omega Y^{(n)}\langle k+1\rangle\right]\right\}
$$

is surjective.

Proof of Theorem 2. We have a fibration $Y\langle k\rangle \rightarrow Y \rightarrow Y^{(k)}$. Taking the $n$th Postnikov section and passing to loop spaces yields the following fibration:

$$
\Omega Y^{(n)}\langle k\rangle \rightarrow \Omega Y^{(n)} \rightarrow \Omega Y^{(k)} .
$$

Applying $[X,-]$, we obtain an exact sequence

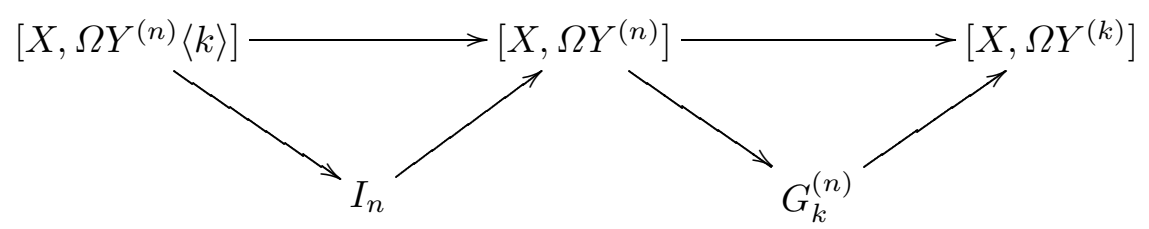

in which $I_{n}$ is the image of $\left[X, \Omega Y^{(n)}\langle k\rangle\right]$ in $\left[X, \Omega Y^{(n)}\right]$. When we take $\lim ^{1}$, we obtain

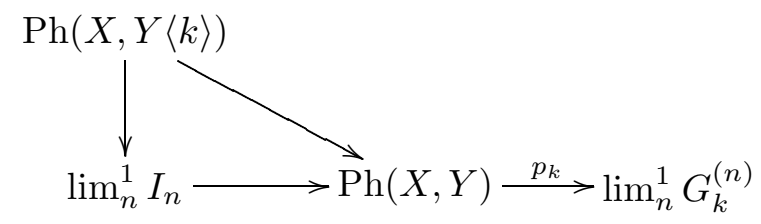

in which the row is exact and the vertical map is surjective. This identifies $\operatorname{Ker}\left(p_{k}\right)$ with $\operatorname{Im}(\operatorname{Ph}(X, Y\langle k\rangle) \rightarrow \operatorname{Ph}(X, Y))$. Therefore $f \sim_{k} *$ if and only if $G^{\prime}(f)>k$. This proves the first statement; the second statement follows from Proposition 1.

Proof of Proposition 3. We have to show that the map $j_{k}: \lim _{n}^{1} G_{k+1}^{(n)} \rightarrow$ $\lim _{n}^{1} G_{k}^{(n)}$ is injective. Consider the short exact sequence of towers

$$
0 \rightarrow\left\{J_{n}\right\} \rightarrow\left\{G_{k+1}^{(n)}\right\} \rightarrow\left\{G_{k}^{(n)}\right\} \rightarrow * .
$$

The fibration

$$
K\left(\pi_{k+1} Y, k\right) \rightarrow \Omega Y^{(k+1)} \rightarrow \Omega Y^{(k)}
$$

shows us that each $J_{n}$ is a quotient of $\left[X, K\left(\pi_{k+1} Y, k\right)\right] \cong H^{k}\left(X ; \pi_{k+1} Y\right)$. If Gray's condition is not satisfied, then this is a finite group. Applying the six-term $\lim _{-1 i m^{1}}$ exact sequence, we obtain the exact sequence

$$
\lim _{n}^{1} J_{n} \rightarrow \lim _{n}^{1} G_{k+1}^{(n)} \rightarrow \lim ^{1} G_{k}^{(n)} \rightarrow * .
$$

Since a tower of finite groups has trivial $\lim ^{1}$ the proof is complete. 
Proof of Theorem 4. According to [2], we can write $A=\operatorname{colim} L_{k}$ where each $L_{k}$ is a subcomplex of $A$ such that $H_{m}\left(L_{k}\right) \rightarrow H_{m}(A)$ is an isomorphism for $m \leq k$ and $H_{m}\left(L_{k}\right)=0$ for $m>k$. A careful examination of the proof reveals that there are subcomplexes $L_{k-1} \subseteq M_{k} \subseteq L_{k}$ so that

(1) $M_{k}$ is a $k$-dimensional subcomplex of $A$,

(2) $H_{m}\left(M_{k} ; \mathbb{Q}\right) \stackrel{\cong}{\longrightarrow} H_{m}(X ; \mathbb{Q})$ for $m \leq k$.

It follows that $A \rightarrow A / M_{k}$ induces an isomorphism in rational homology in dimensions above $k$.

We see from the commutative diagram

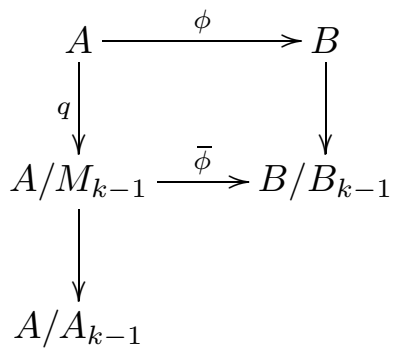

that $\mathrm{Ph}^{k}(A, Y) \subseteq q^{*}\left(\mathrm{Ph}\left(A / M_{k-1}, Y\right)\right)$. Observe also that $\bar{\phi}$ induces surjections $\bar{\phi}^{*}: H^{m}\left(B / B_{k-1} ; \mathbb{Q}\right) \rightarrow H^{m}\left(A / M_{k-1} ; \mathbb{Q}\right)$ for $m \leq l$.

The proof of Theorem 4 now reduces to the following lemma.

Lemma 4.1. If $\phi: A \rightarrow B$ and $\phi^{*}: H^{m}(B ; \mathbb{Q}) \rightarrow H^{m}(A ; \mathbb{Q})$ is surjective for each $m \leq l$, then $\phi$ induces a surjection on $\sim_{l+1}$-equivalence classes.

Proof. Write $G_{k}^{(n)}(A)=\operatorname{Im}\left(\left[A, \Omega Y^{(n)}\right] \rightarrow\left[A, \Omega Y^{(k)}\right]\right)$ and similarly for $G_{k}^{(n)}(B)$. We have to show that $\phi$ induces a surjection $\lim _{n}^{1} G_{l+1}^{(n)}(B) \rightarrow$ $\lim _{n}^{1} G_{l+1}^{(n)}(A)$. To do this, we will show that each map $G_{l+1}^{(n)}(B) \rightarrow G_{l+1}^{(n)}(A)$ rationalizes to a surjection. Since these are finitely generated nilpotent groups, it will follow from Lemma 1.3 of [8] that the images of these maps have finite index. These towers also have the special property that each term has finite index in the next (Proposition 0.1 of [9]). It is proved in Lemma 2.2 of [8] that a map of towers such as these in which the images have finite index induces a surjection on $\lim ^{1}$.

The map $G_{l+1}(B) \rightarrow G_{l+1}(A)$ is by definition $\phi^{*}:\left[B, \Omega Y^{(l+1)}\right] \rightarrow$ $\left[A, \Omega Y^{(l+1)}\right]$. Since these groups only depend on finite skeleta of $A$ and $B$, the rationalization of this map can be identified with $\phi^{*}:\left[B, \Omega Y_{0}^{(l+1)}\right] \rightarrow$ $\left[A, \Omega Y_{0}^{(l+1)}\right]$. Since $\phi$ induces a surjection on rational cohomology, and loop spaces are rationally equivalent to products of Eilenberg-MacLane spaces, this map is a surjection. 
Because of the finite index property of these towers, we know that each map $G_{l+1}^{(n)} \rightarrow G_{l+1}$ is a rational isomorphism. It follows from the commutative diagram

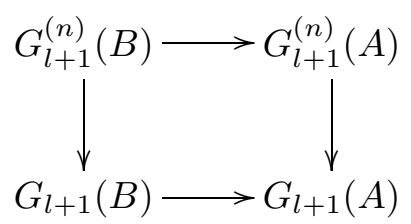

that $G_{l+1}^{(n)}(B) \rightarrow G_{l+1}^{(n)}(A)$ rationalizes to a surjection, as desired.

Proof of Theorem 5. The proof of the dual to Theorem 4 is analogous. The only difference is that the better naturality properties of the Postnikov sections make it unnecessary to fiddle with dual versions of the $M_{k}$.

Proof of Theorem 6. Since $\operatorname{Ph}^{l+1}(A, Y)=\operatorname{Ker}\left(p_{l+1}\right)$, we have to show that $p_{l+1} \phi^{*}\left(\mathrm{Ph}^{k}(B, Y)\right)=*$. Let

$$
A \stackrel{\phi}{\longrightarrow} B \stackrel{\theta}{\longrightarrow} C
$$

be a cofiber sequence. Then $\theta^{*}: H^{m}(C ; \mathbb{Q}) \rightarrow H^{m}(B ; \mathbb{Q})$ is surjective for $k \leq m \leq l$. In the diagram

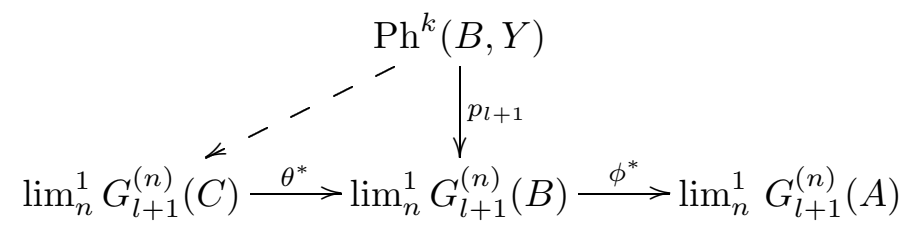

the row is not exact, but the composition is trivial. By Theorem $4, \theta^{*}$ is surjective, so $\phi^{*}$ is trivial.

The proof of the dual result is similar.

Proof of Theorem 8. The groups in the tower $\left\{\left[X,\left(\Omega S^{k+1}\right)^{(m)}\right]\right\}$ are each countable, and the image of each term in the next has finite index by Theorem 0.1 of [9].

First we assume that

$$
\lim _{m}^{1}\left[X, \Omega\left(S^{k+1}\right)^{(m)}\right]=\operatorname{Ph}\left(X, S^{k}\right)=* .
$$

By Theorem 2 of [7], the tower $\left\{\left[X,\left(\Omega S^{k+1}\right)^{(m)}\right]\right\}$ must be Mittag-Leffler. By Lemma 3.2 of [7], this means that the index of

$$
\begin{aligned}
\operatorname{Im}\left(\left[X, \Omega S^{k+1}\right] \rightarrow[X,\right. & \left.\left.\left(\Omega S^{k+1}\right)^{(m)}\right]\right) \\
& =\operatorname{Im}\left(\lim _{m}\left[X, \Omega\left(S^{k+1}\right)^{(m)}\right] \rightarrow\left[X, \Omega\left(S^{k+1}\right)^{(m)}\right]\right)
\end{aligned}
$$

in $\left[X, \Omega\left(S^{k+1}\right)^{(m)}\right]$ is finite for each $m$. Taking $m=k+1$, we see that the index of

$$
\operatorname{Im}\left(\left[X, \Omega S^{k+1}\right] \rightarrow H^{k}(X ; \mathbb{Z})\right)
$$


in $H^{k}(X ; \mathbb{Z})$ is finite. Choose a basis $u_{1}, \ldots, u_{n}$ of integral classes for $H^{k}(X ; \mathbb{Q})$. Then there are maps $g_{i}: X \rightarrow \Omega S^{k+1}$ and constants $\lambda_{i}$ such that

$$
\lambda_{i} u_{i} \in g_{i}^{*}\left(H^{k}\left(\Omega S^{k+1} ; \mathbb{Z}\right)\right) .
$$

It follows that the map $g: X \rightarrow \prod_{i=1}^{n} \Omega S^{k+1}$, given by $g_{i}$ in the $i$ th coordinate, induces a surjection in rational cohomology.

Now assume that such a map $g: X \rightarrow \prod \Omega S^{k+1}$ is given, and let $f \in$ $\mathrm{Ph}^{k}(X, Y)$. Since $g^{*}$ induces a surjection in $k$-dimensional rational cohomology, there is a phantom map $f^{\prime} \sim_{k+1} f$ such that $f^{\prime} \in g^{*}\left(\operatorname{Ph}\left(\prod \Omega S^{k+1}, Y\right)\right)$. But it follows easily from Example 2.3 of [4] that $\operatorname{Ph}\left(\prod \Omega S^{k+1}, Y\right)=*$, which shows that $f \sim_{k+1} *$; in other words, $f \in \mathrm{Ph}^{k+1}(X, Y)$.

Proof of Theorem 9. The proof that condition (1) implies condition (2) is strictly dual to that of Theorem 8 .

Now assume a map $g: \bigvee \Sigma K(\mathbb{Z}, k) \rightarrow Y$ is given which induces a surjection $g_{*}: \pi_{k+1}(\bigvee \Sigma K(\mathbb{Z}, k)) \otimes \mathbb{Q} \rightarrow \pi_{k+1}(Y) \otimes \mathbb{Q}$. Since the inclusion of a wedge into the corresponding product has a section after suspending, we can form the composite

$$
\prod K(\mathbb{Z}, k) \rightarrow \Omega \Sigma\left(\prod K(\mathbb{Z}, k)\right) \rightarrow \Omega \Sigma(\bigvee K(\mathbb{Z}, k)) \stackrel{\Omega g}{\longrightarrow} \Omega Y .
$$

This composite map, $g$, induces a surjection in $\pi_{k}(-) \otimes \mathbb{Q}$.

Consider the diagram

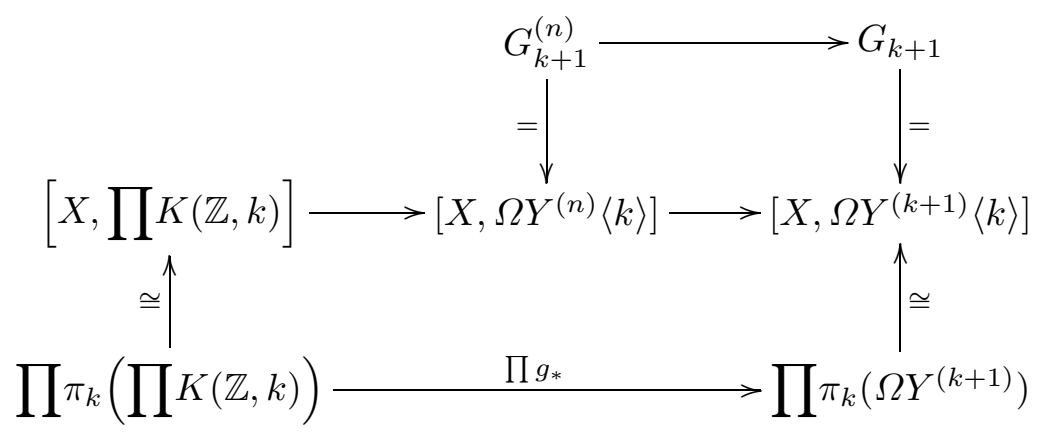

Since the image of $\prod g_{*}$ has finite index, the index of $G_{k+1}^{(n)}$ in $G_{k+1}$ has a finite lower bound for all $n$. This implies that the tower $\left\{G_{k+1}^{(n)}\right\}$ is MittagLeffler, and so

$$
\operatorname{Ph}(X, Y\langle k\rangle)=\mathrm{Ph}^{k+1}(X, Y\langle k\rangle) .
$$

The calculation

$$
\left.\left.\operatorname{Ph}^{k}(X, Y)=\operatorname{Ph}(X, Y\langle k\rangle)\right)=\operatorname{Ph}^{k+1}(X, Y\langle k\rangle)\right) \subseteq \operatorname{Ph}^{k+1}(X, Y)
$$

completes the proof. 
Proof of Theorem 10. Write $G_{n}=\left[X, \Omega Y^{(n)}\right]$, as above. Since $\operatorname{Ph}(X, Y)$ $\cong \lim _{n}^{1} G_{n}$ and $\underline{\mathrm{Ph}}(X, Y) \cong \lim _{k} \lim _{n}^{1} G_{k}^{(n)}$, Theorem 10 a direct consequence of the following algebraic result.

Proposition 10.1. If $\left\{G_{n}\right\}$ is a tower of finitely generated nilpotent groups and $\lim _{k} \lim _{n}^{1} G_{k}^{(n)}=*$, then $\lim _{n}^{1} G_{n}=*$.

Proof. As we have seen, the tower $\left\{\lim _{n}^{1} G_{k}^{(n)}\right\}$ is a tower of surjections. Because $\lim _{k} \lim _{n}^{1} G_{k}^{(n)}=*$, it must be that $\lim _{n}^{1} G_{k}^{(n)}=*$ for all large values of $k$, say for $k \geq N$.

By Theorem 0.1 of [9], each tower $\left\{G_{k}^{(n)}\right\}$ consists of finitely generated nilpotent groups. Since Theorem 2 of [7] tells us that such a tower has trivial $\lim ^{1}$ if and only if it is Mittag-Leffler, we conclude that the tower $\left\{G_{k}^{(n)}\right\}$ is Mittag-Leffler for $k \geq N$. This does not quite imply that the tower $\left\{G_{n}\right\}$ is Mittag-Leffler; but it does show that the tower $\left\{G_{n}^{\prime}\right\}$, which is defined by $G_{n}^{\prime}=G_{n}$ for $n \geq N$ and $G_{n}^{\prime}=0$ otherwise, is Mittag-Leffler. Since these towers have isomorphic $\lim ^{1}, \lim _{n}^{1} G_{n}=*$, as desired.

Proof of Corollary 11. Write $X=\operatorname{colim} M_{k}$, where the $M_{k}$ are as in the proof of Theorem 4. To show that $\mathrm{Ph}^{k+1}(X, Y)=*$, recall that

$$
\mathrm{Ph}^{k+1}(X, Y) \subseteq \operatorname{Im}\left(\operatorname{Ph}\left(X / M_{k}, Y\right) \rightarrow \operatorname{Ph}(X, Y)\right) .
$$

Since $X / M_{k}$ is rationally trivial, Proposition 3 shows that

$$
\operatorname{Ph}\left(X / M_{k}, Y\right)=\mathrm{Ph}^{\infty}\left(X / M_{k}, Y\right)
$$

for every finite type nilpotent $Y$, and so $\operatorname{Ph}\left(X / M_{k}, Y\right)=*$ by Theorem 10 .

The proof of the dual result is similar.

Proof of Theorem 12. In both cases, we have a map of towers of finitely generated abelian groups which induces a surjection on $\lim _{k} \lim _{n}^{1}$, and we want to conclude that the map induces a surjection on $\lim ^{1}$. This is a purely algebraic statement which we prove below.

Proposition 12.1. If $\phi:\left\{B_{n}\right\} \rightarrow\left\{A_{n}\right\}$ is a map of towers of finitely generated abelian groups such that $\phi: \lim _{k} \lim _{n}^{1} B_{k}^{(n)} \rightarrow \lim _{k} \lim _{n}^{1} A_{k}^{(n)}$ is surjective, then $\phi: \lim _{n}^{1} B_{n} \rightarrow \lim _{n}^{1} A_{n}$ is also surjective.

Proof. According to Bousfield and Kan ([1], pp. 254-255), for any tower $\left\{G_{n}\right\}$ there is a short exact sequence

$$
* \rightarrow \lim _{k}^{1} \lim _{n} G_{k}^{(n)} \rightarrow \lim _{n}^{1} G_{n} \rightarrow \lim _{k} \lim _{n}^{1} G_{k}^{(n)} \rightarrow * .
$$

Let $C_{n}$ be the cokernel of $B_{n} \rightarrow A_{n}$. From the six-term exact sequence and the Bousfield-Kan short exact sequence, we obtain the following diagram: 


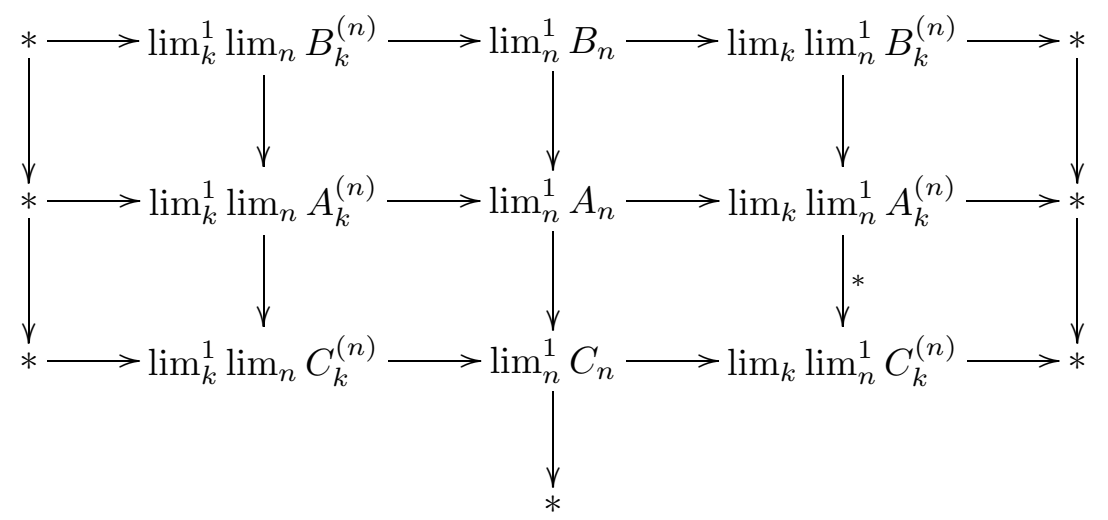

in which the rows and the center column are exact, and each vertical composite is trivial. We have to show that $\lim _{n}^{1} C_{n}=*$. Since the maps

$$
\lim _{n}^{1} A_{n} \rightarrow \lim _{n}^{1} C_{n} \quad \text { and } \quad \lim _{k} \lim _{n}^{1} B_{k}^{(n)} \rightarrow \lim _{k} \lim _{n}^{1} A_{k}^{(n)}
$$

are both surjective, Proposition 10.1 applies to the tower $\left\{C_{n}\right\}$.

\section{References}

[1] A. K. Bousfield and D. M. Kan, Homotopy Limits, Completions and Localizations, Lecture Notes in Math. 304, Springer, Berlin, 1972.

[2] E. H. Brown, Jr. and A. H. Copeland, Jr., An homology analogue of Postnikov systems, Michigan J. Math. 6 (1959), 313-330.

[3] B. Gray, Operations and a problem of Heller, Ph.D. thesis, Univ. of Chicago, 1965.

[4] B. Gray and C. A. McGibbon, Universal phantom maps, Topology 32 (1993), 371394.

[5] Lê Minh Hà, On the Gray index of phantom maps, Ph.D. thesis, Wayne State Univ., 2000.

[6] C. A. McGibbon, Phantom maps, Chapter 25 in: Handbook of Algebraic Topology, Elsevier, 1995.

[7] C. A. McGibbon and J. M. Møller, On spaces with the same $n$-type for all $n$, Topology 31 (1992), 177-201.

[8] C. A. McGibbon and J. Roitberg, Phantom maps and rational equivalences, Amer. J. Math. 116 (1994), 1365-1379.

[9] C. A. McGibbon and R. Steiner, Some questions about the first derived functor of the inverse limit, J. Pure Appl. Algebra 103 (1995), 325-340.

[10] C. A. McGibbon and J. Strom, Numerical invariants of phantom maps, to appear.

[11] R. J. Milgram, Surgery with coefficients, Ann. of Math. 100 (1974), 194-248.

[12] G. W. Whitehead, Elements of Homotopy Theory, Grad. Texts in Math. 61, Springer, 1978. 
[13] A. Zabrodsky, On phantom maps and a theorem of H. Miller, Israel J. Math. 58 (1987), 129-143.

Department of Mathematics

Department of Mathematics

University of Toledo

Dartmouth College

Toledo, OH 43606, U.S.A.

Hanover, NH 03755, U.S.A.

E-mail: lha@math.utoledo.edu

E-mail: jeffrey.strom@dartmouth.edu

Received 23 November 1999;

in revised form 9 October 2000 